of salt-fed transgenic animals expressing a mutated form of Ncx1.3 that lacked the SEA0400 binding site. Finally, mice heterozygous for the NCX1 mutation had low salt sensitivity.

So much for calcium ion entry. The authors next turned to an examination of sodium ions. It is known that elevated local levels of sodium trigger vasoconstriction. Cardiotonic steroids such as endogenous ouabain (related to digitalis) that inhibit the plasma membrane $\mathrm{Na}^{+} / \mathrm{K}^{+}$-ATPase are elevated in about $50 \%$ of patients with essential hypertension and in several salt-sensitive hypertensive animal models ${ }^{7}$. In vascular smooth muscle, the inhibition of $\mathrm{Na}^{+} / \mathrm{K}^{+}$ATPases by ouabain increases local $\mathrm{Na}^{+}$ levels. The authors found that SEA0400 abolished the effects induced by ouabain, indicating that NCX1.3 functions downstream from $\mathrm{Na}^{+} / \mathrm{K}^{+}$-ATPase. As a result, in the presence of ouabain, NCX1 raises the cytoplasmic levels of calcium and initiates vasoconstriction. It seems that by implicating the NCX1.3 transporter in salt-dependent hypertension, the authors have identified the elusive link between local sodium ion levels and the contraction of the vascular smooth muscle (Fig. 1$)^{8}$.

What does all this mean for normal physiological conditions? According to Iwamoto et al. the calcium entry mode of NCX1 in vascular smooth muscle has no effect on blood pressure under normal conditions. NCX1heterozygous mice maintain normal blood pressure and SEA0400 does not affect arterial blood pressure in normotensive animals. Furthermore, intrafemoral infusion of SEA0400 in normal rats and dogs does not change arterial blood flow unless the arteries are perfused with exogenous ouabain. Why, then, does this system exist? The experiments of Iwamoto et al. suggest that NCX1 is functionally linked to ouabain. Ouabain seems to be necessary for NCX1-mediated vasoconstriction. Since endogenous ouabain is elevated in blood upon increased $\mathrm{Na}^{+}$uptake, hypoxia and physical exercise, vascular NCX1 may be a key mediator of blood pressure regulation under stress conditions.

Where are we now in understanding salt and hypertension? Perhaps the most significant finding in the new study is that the vascular $\mathrm{Na}^{+} / \mathrm{Ca}^{2+}$ exchanger modulates blood pressure. Thus, excessive salt intake, a major risk factor for hypertension, may now be directly linked to cytosolic calcium elevation and vasoconstriction of arteries.

Before NCX1 becomes a target for a new class of antihypertensive drugs, it is important to understand exactly how NCX1 works, and whether it helps to control blood pres- sure in humans. Since NCX1 exists in multiple splice isoforms throughout the body-for instance, the molecule is present in the heart and brain - it will be important to investigate the effect of such pharmacologic agents on these organs.

That excessive salt intake can increase blood pressure is now well established in animal models and humans. Whether decreasing daily salt intake is of benefit to the general public is, however, still a contentious issue. Hence, determining which patients will benefit from a low-salt diet is a primary goal in modern hypertension research. The study by Iwamoto et al. introduces a new factor that may be diagnostic for salt-sensitive hypertension. As we wait for the story to unravel, perhaps it is worth emphasizing the wisdom of Huang Ti Nei Ching and promote prevention with a grain of salt.

1. O'Shaughnessy, K.M \& Karet, F.E. J. Clin. Invest 113, 1075-1081 (2004).

2. Lifton, R.P. et al. Cell 104, 545-556 (2001).

3. Iwamoto, T. et al. Nat. Med. 10, 1193-1199 (2004).

4. Wakimoto, K. et al. J. Biol. Chem. 275, 36991-36998 (2000).

5. Krushkal, J. et al. Circulation 99, 1407-1410 (1999).

6. Iwamoto, T. et al. J. Biol. Chem. 279, 7544-7553 (2004).

7. Hamlyn, J.M. et al. J. Hypertens. 14, 523-533 (1996).

8. Blaustein, M.P. \& Lederer, W.J. Physiol Rev. 3, 763-854 (1999).

\title{
H. pylori's inside job
}

Helicobacter pylori, like many other pathogenic bacteria, inserts substances into human cells that can modify cell behavior. Jérôme Viala et al. now find that human cells have turned this system against the bacterium. Delivery of the bacterial substance peptidoglycan alerts the cell to the presence of $H$. pylori, setting in motion antibacterial defenses (Nature Immunology 5, 1166-1174). (Shown is $H$. pylori infecting human cells; peptidoglycan is in purple and individual bacteria appear as refractile objects).

The authors began with the question: how does the host recognize H. pylori? Previous researchers had ruled out some suspects, such as Toll-like receptors, which recognize conserved bacterial components (see p. 1175). The authors turned their attention to another set of proteins that recognize bacteria, the Nod proteins, and found that human cells needed Nod 1 to mount an inflammatory response to $H$. pylori. There was one problem with this scenario, however: Nod proteins are expressed inside cells, and H. pylori is an extracelluar pathogen.

The type IV secretion system gets around this issue. The researchers provide evidence that the system delivers peptidoglycan, which Nod1 recognizes, into the cell. This recognition sets in motion an inflammatory response that can lead to tissue damage. The type IV secretion system is encoded on a region of bacterial DNA - the Cag pathogenicity islandwhich is present in $\mathrm{H}$. pylori strains that tend to be associated with more severe forms gastric inflammation, ulceration and cancer.

Charlotte Schubert

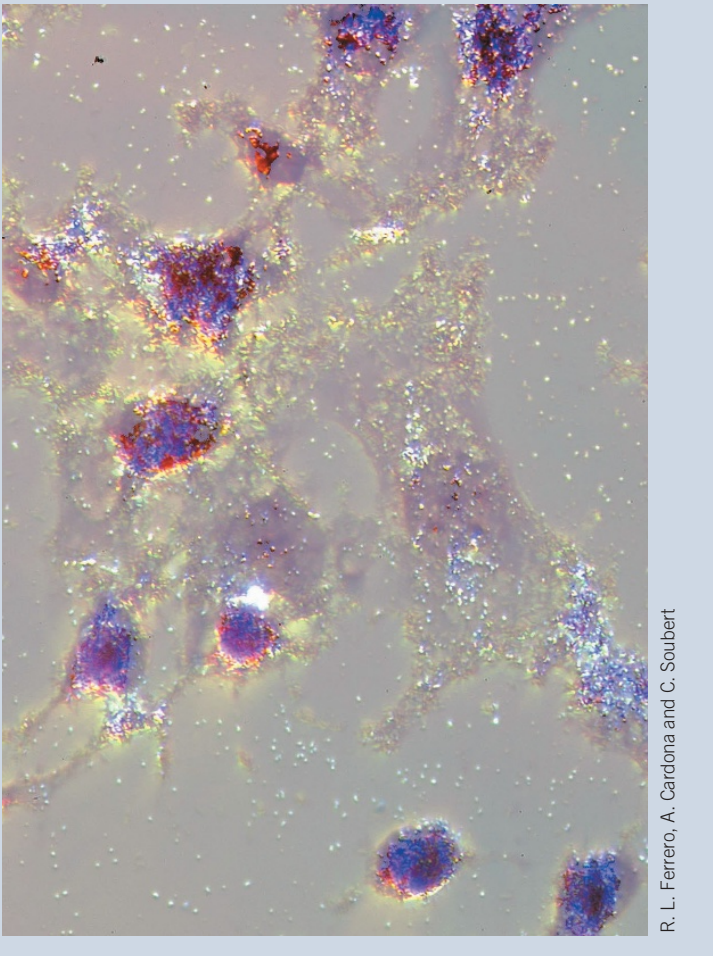

